\title{
Association between shortage of energy supply and nuclear gene mutations leading to carcinomatous transformation (Review)
}

\author{
JIANPING DU
}

Department of Oncology, Anhui Provincial Hospital Affiliated to Anhui Medical University, Hefei, Anhui 230001, P.R. China

Received July 24, 2015; Accepted September 18, 2015

DOI: $10.3892 / \mathrm{mco} .2015 .656$

\begin{abstract}
Anaerobic bacteria use glycolysis, an oxygen-independent metabolic pathway, whereas energy metabolism in the evolved eukaryotic cell is performed via oxidative phosphorylation, with all eukaryotic cell activities depending upon high energy consumption. However, in cancer cells evolving from eukaryotic cells, the energy metabolism switches from oxidative phosphorylation to glycolysis. The shortage of energy supply induces cancer cells to acquire specific characteristics. Base pair renewal is the most energy-consuming process in the cell, and shortage of energy supply may lead to errors in this process; the more prominent the shortage in energy supply, the more errors are likely to occur in base pair renewal, resulting in gene mutations and expression of cancer cell characteristics. Thus, shortage of energy supply is associated with carcinomatous transformation.
\end{abstract}

\section{Contents}

1. Introduction

2. Endosymbiotic theory and gene alterations

3. Prokaryote hypothesis and the Warburg effect

4. Energy metabolism determines gene status

5. Conclusion

\section{Introduction}

Metastasis occurs when cancer cells disseminate from the primary tumor and form secondary tumors at distant sites in the body. This is a crucial characteristic that distinguishes the cancer cell from the normal parent cell; all types of cancer cells differ from their normal parent cells to various degrees. Investigating the underlying mechanism may help identify

Correspondence to: Dr Jianping Du, Department of Oncology, Anhui Provincial Hospital Affiliated to Anhui Medical University, 9 Lujiang Road, Hefei, Anhui 230001, P.R. China

E-mail: djppk@163.com

Key words: base pair renewal, cancer cell, energy metabolism, gene mutation, glycolysis, oxidative phosphorylation more effective ways to treat cancer patients. The commonly accepted theory is that genes determine cell characteristics, and there is a hypothesis that mitochondrial oncogenesis is the trigger of gene mutations leading to the formation of cancer cells from normal cells (1). As the cancer cell is considered to evolve from the eukaryote, the answer may lie with the process of eukaryotic cell evolution and normal somatic cell oncogenesis.

\section{Endosymbiotic theory and gene alterations}

The endosymbiotic theory was proposed by Lynn Margulis in the 1960s and suggested that aerobic bacteria (ancient mitochondria) were ingested by anaerobic bacteria (ancient prokaryotic cells) and transferred most of their DNA to the nucleus of the anaerobic bacteria; in this manner, they both had a survival advantage for as long as they continued their 'partnership'. The aerobic bacteria utilized the oxygen, which is toxic for anaerobic bacteria, whereas the anaerobic bacteria ingested nutrients and protected the aerobic 'symbiote' $(2,3)$. This theory provided a satisfactory explanation for the evolution of eukaryotic cells. With continued aerobic metabolism, a reasonable conclusion may be drawn that the ancient genes regulating pathway signaling in glucolysis were obliged to mutate (alter themselves) to survive under the conditions of oxidative phosphorylation. The result was that the genes of the eukaryotic cell (wild-type genes) were also available in another status (mutant genes) if the eukaryotic cell was under atavistic conditions. As eukaryotic cells degenerate prokaryotic cells the related genes for prokaryotic cells would be expressed.

\section{Prokaryote hypothesis and the Warburg effect}

In light of the endosymbiotic theory and the hypothesis that the replicative ability of cancer cells is similar to the proliferation of prokaryotic cells, Klaus Grossgebauer first proposed the prokaryote hypothesis of oncogenesis, suggesting that a re-evolution from eukaryotic to prokaryotic cells leads to cancer development $(4,5)$. This theory supported that, under special conditions, such as oncogenesis, eukaryotic cells may express certain prokaryotic cell characteristics. The Warburg effect is a typical example of this re-evolution, which is reflected in the metabolism.

Under physiological conditions, the majority of normal eukaryotic cells use the more energy-efficient oxidative 
phosphorylation as the main route to generate ATP. However, Warburg observed that cancer cells performed energy metabolism in a manner that was different from normal adult cells; cancer cells obtained energy through glycolysis rather than oxidative phosphorylation. Therefore, Warburg formulated the hypothesis that the cause of cancer was primarily the defect in energy metabolism (6,7). A number of cancer cells have been proven to actively use the glycolytic pathway for ATP generation, even in the presence of oxygen (aerobic glycolysis). It may be concluded that energy metabolism determines the development of cancer, in the sense that chain reactions directed to eukaryotic cells are initiated if oxidative phosphorylation plays a dominant role; likewise, chain reactions directed to cancer cells are initiated if glucolysis plays a dominant role. However, the mechanism underlying the induction of these alterations has not been fully elucidated.

\section{Energy metabolism determines gene status}

As mentioned above, our hypothesis was based on the assumption that the base pairs of genes are continually renewed during the life cycle of cells, maintaining a dynamic balance between reproduction and extinction; it is also based on the fact that each cell characteristic is affected by multiple genes and the majority of the genes determines the characteristics of the cells.

The process leading to genes expressing characteristics of eukaryotic cells is based on adequate energy supply. With eukaryotic cell evolution, a single eukaryotic cell gives rise to multiple eukaryotic cells, with the daughter cells maintaining the same shape as the mother cell. With multi-eukaryotic cell evolution, an advanced differentiation occurs (development of advanced organisms), meaning that certain advanced genes evolve to regulate the structure of evolved eukaryotic cells (the process during which ancient genes evolve to advanced genes is identical to the one during which the base pairs of chromosomes are repermutated and recombined). It may be hypothesized that this progression occurs under appropriate conditions of oxidative phosphorylation, and that the genes expressing the characteristics of eukaryotic cells are transformed into the dominant genes based on adequate energy supply (normal expressions of eukaryotic cell characteristics depend upon the genes of eukaryotic cells, which include the base pairs encoding the normal eukaryotic cell characteristics and require a high energy supply). It has been proven that a high ATP level is required for DNA synthesis (8) and DNA stabilization, which maintains the characteristics of eukaryotic cells.

Shortage of energy supply alters the base pairs of genes. As soon as malignant transformation of eukaryotic cells occurs, cancer cells have to obtain energy through glucolysis rather than oxidative phosphorylation. The total energy supply is rapidly reduced, as the total energy yield of glucolysis is different. The glucolytic pathway for ATP generation is unable to produce sufficient energy for the normal renewal of the base pairs of eukaryotic cell genes. At this time, normal base pairs are replaced by abnormal base pairs, resulting in gene mutations, DNA methylation, overexpression and no or reduced expression (9-12). Different chromosomes exhibit different abilities to tolerate the shortage of energy, with some chromosomes being more vulnerable compared with others. For this reason, the proteins of cancer cells depend upon the changes in the quantity of base pairs, which determine the degree of gene changes in the chromosomes.

Abnormal genes express characteristics of cancer cells. It has been proven that a cell characteristic depends upon a number of proteins, which are encoded by a set of genes. The abnormal gene is unable to produce a protein identical to that encoded by the primary normal gene. Therefore, the more genes change, the more cancer cell proteins deviate from parent-cell proteins and the more characteristics of cancer cells resemble those of ancient prokaryotic cells.

\section{Conclusion}

Following malignant transformation of eukaryotic cells, the cancer cells cannot obtain sufficient energy from glycolysis to maintain normal chromosome base pair renewal. Abnormal base pairs replace normal base pairs, inducing gene mutations. The more genes change, the more abnormal proteins are produced, which cause the characteristics of cancer cell to resemble those of ancient prokaryotic cells.

\section{References}

1. Du J: Hypothesis of mitochondrial oncogenesis as the trigger of normal cells to cancer cells. Med Hypotheses 82: 744-747, 2014.

2. Sagan L: On the origin of mitosing cells. J Theor Biol 14: 255-274, 1967.

3. Margulis L: Origin of eukaryotic cells, Yale University Press, New Haven, Connecticut 1970.

4. Grossgebauer K: Cancer: Re-evolution of eukaryotic to prokaryotic replication units - a result of genetic transposition? Biosystems 16: 253-258, 1984.

5. Grossgebauer K: Prokaryotic mechanisms in eukaryotes: Experimental data and speculations. Med Hypotheses 28: 219-223, 1989.

6. Warburg O: The Metabolism of Tumors. Arnold Constable, London, 1930.

7. Warburg O: On the origin of cancer cells. Science 123: 309-314, 1956.

8. Enomoto K, Tanuma S and Yamada M: ATP requirement for the processes of DNA replication in isolated HeLa cell nuclei. J Biochem 89: 801-807, 1981

9. Scoccianti C, Vesin A, Martel G, Olivier M, Brambilla E, Timsit JF, Tavecchio L, Brambilla C, Field JK and Hainaut P; European Early Lung Cancer Consortium: Prognostic value of TP53, KRAS and EGFR mutations in nonsmall cell lung cancer: The EUELC cohort. Eur Respir J 40: 177-184, 2012.

10. Karsli-Ceppioglu S, Dagdemir A, Judes G, Ngollo M, Penault-Llorca F , Pajon A, Bignon YJ and Bernard-Gallon D: Epigenetic mechanisms of breast cancer: An update of the current knowledge. Epigenomics 6: 651-664, 2014

11. Mosalpuria K, Hall C, Krishnamurthy S, Lodhi A, Hallman DM, Baraniuk MS, Bhattacharyya A and Lucci A: Cyclooxygenase-2 expression in non-metastatic triple-negative breast cancer patients. Mol Clin Oncol 2: 845-850, 2014.

12. Wang W, Chen L, Tang Q, Fan Y, Zhang X and Zhai J: Loss of ARHI expression in colon cancer and its clinical significance. Contemp Oncol (Pozn) 18: 329-333, 2014. 